following vaccination. 79\% routinely recall men for Hepatitis B vaccination.

Discussion/conclusion There is evidence of variation in clinical practice between clinics in the UK, not all of which can be explained by variations in local epidemiology and some of which has significant cost implications. Results have generated debate in the MSM SIG on the rationale for local policies.

\section{P016 BASHH MSM SIG CLINIC SURVEY; HOLISTIC AND INCLUSIVE CARE}

${ }^{1}$ Dan Clutterbuck, ${ }^{2}$ Lisa McDaid*, ${ }^{3}$ MSM Special Interest Group. ${ }^{1}$ Chalmers Centre, Edinburgh, UK; ${ }^{2} M R C / C S O$ Social \& Public Health Sciences Unit, University of Glasgow, Glasgow, UK; ${ }^{3} B A S H H$, London, UK

\subsection{6/sextrans-2016-052718.71}

Background/introduction MSM experience a disproportionate burden of ill health in relation to sexual health, mental health and substance use.

Aim(s)/objectives Our aim was to investigate practice across the UK in aspects of the clinical care of MSM where evidence is absent, or guidance varies.

Methods An online questionnaire was distributed to BASHH, FSRH members and CSP audit sites for one month to $31^{\text {st }}$ October 2015. Questions covered assessment of risk factors for STIs and HIV and other elements of holistic care.

Results There were 149 complete responses. A written policy on obtaining a history of alcohol or recreational drug use was reported by $62 \%$ and $66 \%$ of respondents respectively. $58 \%$ and $57 \%$ had a documented pathway for alcohol or drug use problems. $67 \%$ had dedicated services for behaviour change interventions, but only $20 \%$ had dedicated sexual dysfunction services. HPV vaccination and PrEP were provided in some form (including with charges, or by private prescription) by $13 \%$ of clinics, but $58 \%$ and $45 \%$ reported no local discussion yet on HPV vaccination or PrEP for MSM. Support for local CaSH \& Youth services in providing care for MSM was given through a formal arrangement or MCN by $30 \%$ of respondents and informally by a further $47 \%$.

Discussion/conclusion There is considerable variation in the breadth of clinical holistic care offered across the UK, suggesting missed opportunities to address the interrelated health inequalities experienced by MSM. GUM clinics may be under-utilised as a source of local expertise in the care of MSM.

\section{P017 ARE WE MISSING OPPORTUNITIES? - A RETROSPECTIVE AUDIT ON LATE DIAGNOSIS OF HIV}

Madhusree Ghosh*, Adrian Palfreeman. University Hospitals of Leicester, Leicester, UK

\subsection{6/sextrans-2016-052718.72}

Introduction $24 \%$ of deaths among HIV-positive adults in the UK are due to late diagnosis of HIV. Many 'late presenters' have previously been seen by healthcare professionals and the diagnosis missed. The study city also has a significantly higher late diagnoses rate $(61 \%)$ compared to the national rate $(45 \%)$.

Aim To identify: newly- diagnosed HIV positive patients between 2010- 2012; rates of 'late' diagnosis; missed opportunities for testing.
Methods Reviewing the case-notes of all newly diagnosed HIV positive residents in the study city, with a CD4 count $<350$ cells/ $\mu$ l.

National Testing guidelines were used to define the indicators for testing. The primary outcome was 'late' diagnosis (CD4 count $<350$ cells $/ \mu \mathrm{l})$. The secondary outcome was 'missed opportunity'(failure to diagnose HIV within one month) in the presence of an indicator for testing.

Results From 180 new HIV-positive cases reviewed 85 met the case definition of 'late' diagnosis, 38 of which had been diagnosed prior to 2010. Meaning the true number of late diagnosis cases during the audit period was 47 (26\%). 14.8\% of cases had pre-existing HIV indicators, and $46 \%$ of cases had missed opportunities for early diagnosis.

Conclusions This audit demonstrates that the actual late-diagnosis rate is lower than that reported previously. There is a high rate of missed opportunities, which warrants increasing the awareness of clinicians and the general population for early detection of HIV, the responsibility for which rests with both clinicians and commissioners.

\section{P018 EVOLVING CONTRACEPTIVE OPTIONS IN INDIGENOUS COMMUNITIES IN PANAMA}

${ }^{2,1}$ Alyssa Jacob* ${ }^{1}$ Benjamin LaBrot, ${ }^{3,1}$ Rachel Wallice, ${ }^{4,1}$ Megan Lewis. ${ }^{1}$ Floating Doctors, Bocas del Toro, Panama; ' University Hospitals Bristol NHS Foundation Trust, Bristol, UK; ${ }^{3}$ Swansea University, Swansea, UK; ${ }^{4}$ University of San Francisco, San Francisco, USA

\subsection{6/sextrans-2016-052718.73}

Background Use of contraception in Panamanian indigenous groups is significantly lower than in the general population. Birth-rates in the Ng-be-Buglé group are the highest in the country. A large proportion of the $\mathrm{Ng}$-be-Buglé live in isolated, rural communities with limited sanitation and education facilities and poor access to local health services. A non-governmental organisation (NGO) has been providing primary healthcare to these communities since 2011 and introduced the contraceptive injection in 2013.

Aims To assess the level and trends of contraceptive injection use and to identify associated challenges.

Methods An observational study of depot medroxyprogesterone acetate (DMPA) use in women attending the NGO clinics was carried out.

Results 143 women from 16 communities have used DMPA from the NGO; $46.9 \%$ started in the last 6 months. The most common reason for commencing is family completion. Average age at commencing is 27.6 years (range 12-46) and number of children is 4 (range $0-14$ ). Since starting, 13.3\% have discontinued use and $25.9 \%$ have missed their most recent dose. Missed doses are commonly due to clinic non-attendance. Influence from spouses and misconceptions regarding side-effects are key factors in discontinuation.

Discussion Ng-be-Buglé communities are experiencing unsustainable population growth. Contraceptive options available to these communities remain limited. Despite a recent surge in the uptake of DMPA from the NGO, major challenges regarding long-term use and compliance remain. Our ongoing work aims to broaden contraceptive options for these people and includes implementation of a pilot study introducing the contraceptive implant. 